\title{
Is there a need for yet another staging system for differentiated thyroid cancer?
}

\author{
Michael Bouvet
}

Received: 29 January 2014/ Accepted: 3 February 2014/Published online: 16 February 2014

(c) Springer Science+Business Media New York 2014

Papillary thyroid carcinoma and follicular thyroid carcinoma together comprise a generally indolent group of cancers known as differentiated thyroid carcinomas (DTCs). In 2014 , there will be an estimated 62,980 new cases of differentiated thyroid cancer, and the incidence is rising faster than any other cancer in the USA [1]. Although the prognosis of DTC is generally good, a small subset of patients eventually die of the disease and up to $30 \%$ of patients experience a recurrence of their disease after 30 years of follow-up [2]. As a result, a number of studies have identified various clinical and pathologic predictors for DTC and devised risk-group stratification or staging systems to select those at high risk of disease recurrence for more aggressive surgical and adjuvant treatment, while those at low risk would be spared aggressive treatment.

The American Joint Committee on Cancer (AJCC)/ Union for International Cancer Control tumor-nodemetastasis (TNM) staging system is the most widely used and is considered the international gold standard and the staging system currently recommended by the American Thyroid Association [3]. Other thyroid cancer staging systems include the Mayo Clinic's metastases, age, complete resection, invasion, size [4]; the Lahey Clinic's age, metastases, extent, size [5]; and the European Organization for Research and Treatment of Cancer systems [6]. One review of the literature found over 14 different staging systems for papillary thyroid cancer alone [7].

\footnotetext{
M. Bouvet

Department of Surgery, University of California San Diego, San Diego, CA, USA

M. Bouvet $(\square)$

Moores UCSD Cancer Center, 3855 Health Sciences Drive

\#0987, La Jolla, CA 92093-0987, USA

e-mail: mbouvet@ucsd.edu
}

With so many staging systems for thyroid cancer, one might ask, is there a need for yet another staging system? While the TNM stratification has been found useful at stratifying patients with DTC into prognostic risk groups, it is cumbersome to implement clinically given the large number of bins within this system and the complicated system of arriving at stage information. In 2009, Onitilo et al. [8] reported a simplified quantitative alternative to the TNM system (QTNM) and compared this with the conventional system. Six hundred and fourteen cases of DTC managed at their institution from 1987 to 2006 were identified. Cancerspecific survival (CSS) and disease-free survival (DFS) were calculated by the Kaplan-Meier method, and a simplified QTNM score was devised using a Cox proportional hazards model. The TNM system was quantified as follows: 4 points each for age older than 45 years and presence of neck nodal metastases while 6 points for tumor size larger than $4 \mathrm{~cm}$ or extrathyroidal extension and 1 point for non-papillary DTC. A sum of 0-5 points was low risk, 6-10 points intermediate, and 11-15 points high risk. Comparison with the conventional TNM system and two other systems revealed similar or better discrimination with the QTNM, and this discrimination was maintained when this risk stratification was applied to a unique validation set. The authors concluded that the QTNM system as opposed to the conventional TNM system was a simple and effective method for risk stratification for both recurrence and cancer-specific mortality.

In this issue of Endocrine, Mankarios et al. [9] used an Australian state database to test the validity of the QTNM staging system for assessing prognosis of DTC. Using the Queensland Cancer Registry (QCR), they identified 788 cases of DTC diagnosed from 1982 to 2006 with complete staging information. Causes of death were ascertained by linking the QCR database with the Australian National Death Index. Subjects were staged according to AJCC TNM 
seventh edition and QTNM, and CSS was calculated by the Kaplan-Meier method. Cancer-specific mortality was observed in $22(2.8 \%)$ patients, with 10-year CSS for the cohort of $97.0 \%$ at a median follow-up of 262.8 months. QTNM stage-specific cancer survival at 10 years was 99.6, 97.0, and $78.6 \%$ for low-, intermediate-, and high-risk groups, respectively. This was comparable to the original US dataset in which the QTNM was initially studied, and it fared better at discriminating survival than the standard TNM system, where there was overlap in survival between stages. This study validates the QTNM system in an Australian cohort and shows at least equivalent discriminatory capacity to the current TNM staging system.

There are some limitations to this study that are recognized by the authors. Namely, the Queensland registry had no information on recurrence, and therefore, DFS could not be assessed in this cohort. Likewise, there was an absence of data on the extent of surgery and postsurgical remnant ablation with radioactive iodine. One way to validate QTNM system would be to merge the data from three continents and analyze for CSS (and DFS if possible). This would make a more convincing case that QTNM is more accurate than TNM (or other systems). Lastly, QTNM may not be the appropriate mnemonic as " $\mathrm{M}$ " is excluded and histopathology is included. Ultimately, the QTNM staging system for thyroid cancer will need to be implemented in prospective manner to ensure that all data variables are ascertained and that patient outcome is validated over time.

While no staging system is perfect, the QTNM does appear to have some advantages compared to the TNM. It clearly discriminates between prognostic groups, has clinical relevance, and is simple to use. Only time will tell as to whether the QTNM system will be embraced by the thyroid cancer community. So what does the future hold for the staging thyroid cancer? It is probable that new molecular prognostic variables for thyroid cancer will emerge in this era of personalized medicine. Certain variables, such as BRAF mutations [10], may be incorporated into current staging systems if they provide additional prognostic value and if a general consensus can be obtained regarding their use.

Conflict of interest The author has no conflict of interests to declare.

\section{References}

1. R. Siegel, J. Ma, Z. Zou, A. Jemal, CA Cancer statistics. Cancer J. Clin. 64(1), 9-29 (2014). doi:10.3322/caac.21208

2. J.A. Sipos, E.L. Mazzaferri, The therapeutic management of differentiated thyroid cancer. Expert. Opin. Pharmacother. 9(15), 2627-2637 (2008). doi:10.1517/14656566.9.15.2627

3. American Thyroid Association Guidelines Taskforce on Thyroid, N., Differentiated Thyroid, C., Cooper, D.S., Doherty, G.M., Haugen, B.R., Kloos, R.T., Lee, S.L., Mandel, S.J., Mazzaferri, E.L., McIver, B., Pacini, F., Schlumberger, M., Sherman, S.I., Steward, D.L., Tuttle, R.M.: Revised American Thyroid Association management guidelines for patients with thyroid nodules and differentiated thyroid cancer. Thyroid 19(11), 1167-1214 (2009). doi:10.1089/thy.2009.0110

4. I.D. Hay, C.S. Grant, W.F. Taylor, W.M. McConahey, Ipsilateral lobectomy versus bilateral lobar resection in papillary thyroid carcinoma: a retrospective analysis of surgical outcome using a novel prognostic scoring system. Surgery 102(6), 1088-1095 (1987)

5. B. Cady, R. Rossi, An expanded view of risk-group definition in differentiated thyroid carcinoma. Surgery 104(6), 947-953 (1988)

6. D.P. Byar, S.B. Green, P. Dor, E.D. Williams, J. Colon, H.A. van Gilse, M. Mayer, R.J. Sylvester, M. van Glabbeke, A prognostic index for thyroid carcinoma. A study of the E.O.R.T.C. Thyroid Cancer Cooperative Group. Eur. J. Cancer 15(8), 1033-1041 (1979)

7. B.H. Lang, C.Y. Lo, W.F. Chan, K.Y. Lam, K.Y. Wan, Staging systems for papillary thyroid carcinoma: a review and comparison. Ann. Surg. 245(3), 366-378 (2007). doi:10.1097/01.sla. $0000250445.92336 .2 \mathrm{a}$

8. A.A. Onitilo, J.M. Engel, C.I. Lundgren, P. Hall, L. Thalib, S.A. Doi, Simplifying the TNM system for clinical use in differentiated thyroid cancer. J. Clin. Oncol. 27(11), 1872-1878 (2009). doi: $10.1200 / J C O .2008 .20 .2382$

9. D. Mankarios, P. Baade, P. Youl, R.H. Mortimer, A.A. Onitilo, A. Russell, S.A. Doi, Validation of the QTNM staging system for cancer-specific survival in patients with differentiated thyroid cancer. Endocrine (2013). doi:10.1007/s12020-013-0078-9

10. M. Xing, A.S. Alzahrani, K.A. Carson, D. Viola, R. Elisei, B. Bendlova, L. Yip, C. Mian, F. Vianello, R.M. Tuttle, E. Robenshtok, J.A. Fagin, E. Puxeddu, L. Fugazzola, A. Czarniecka, B. Jarzab, C.J. O'Neill, M.S. Sywak, A.K. Lam, G. RiescoEizaguirre, P. Santisteban, H. Nakayama, R.P. Tufano, S.I. Pai, M.A. Zeiger, W.H. Westra, D.P. Clark, R. Clifton-Bligh, D. Sidransky, P.W. Ladenson, V. Sykorova, Association between BRAF V600E mutation and mortality in patients with papillary thyroid cancer. JAMA 309(14), 1493-1501 (2013). doi:10.1001/ jama2013.3190 